

\title{
Les effets de la Proximité sur l'exécution des politiques publiques: L'exemple d'un Centre de Lutte contre le Cancer (CLCC)
}

\author{
Laurent Mériade, Benoit Nautré, Corinne Rochette, Damien Talbot
}

\section{To cite this version:}

Laurent Mériade, Benoit Nautré, Corinne Rochette, Damien Talbot. Les effets de la Proximité sur l'exécution des politiques publiques: L'exemple d'un Centre de Lutte contre le Cancer (CLCC). Politiques et Management public, 2017, 34 (1/2), pp.147-166. 10.3166/pmp.34.2017.0008 . hal02327098

\section{HAL Id: hal-02327098 \\ https://hal.uca.fr/hal-02327098}

Submitted on 22 Oct 2019

HAL is a multi-disciplinary open access archive for the deposit and dissemination of scientific research documents, whether they are published or not. The documents may come from teaching and research institutions in France or abroad, or from public or private research centers.
L'archive ouverte pluridisciplinaire HAL, est destinée au dépôt et à la diffusion de documents scientifiques de niveau recherche, publiés ou non, émanant des établissements d'enseignement et de recherche français ou étrangers, des laboratoires publics ou privés. 


\title{
Les effets de la Proximité sur l'exécution des politiques publiques : L'exemple d'un Centre de Lutte contre le Cancer (CLCC)
}

\author{
MERIADE L., NAUTRÉ B., ROCHETTE C., TALBOT D., \\ CRCGM \\ Université de Clermont Auvergne \\ Politiques et Management Public, 34/1-2, 2017, pp. 147-166
}

\section{Résumé}

Les principes du nouveau management public infusent progressivement le secteur hospitalier, un secteur marqué par de profondes transformations. La manière dont s'expriment les politiques publiques dans ces établissements permet de mettre en évidence des injonctions paradoxales que les acteurs œuvrant en leur sein tentent de concilier. La mobilisation de la grille de la proximité apporte une lecture originale des manières dont les politiques publiques sont exécutées pour notamment faire face à ces nouveaux défis. Les dimensions de la proximité sont utilisées ici pour étudier la confiance entre les acteurs, le contrôle organisationnel et la légitimité des politiques publiques. La recherche porte sur l'étude du cas d'un Centre de Lutte Contre le Cancer (CLCC). Cette étude croise des données secondaires (comptes rendus de réunion, présentation du plan stratégique du Centre, organigramme, rapports de gestion) et des entretiens semi-directifs auprès de personnels des quatre catégories les plus représentatives du personnel hospitalier (dirigeants, médecins, personnels de soin, personnel des fonctions support).

$\underline{\text { Mots clés }}$ : Politiques publiques, proximités, confiance, contrôle, légitimité.

\section{Introduction}

Le secteur hospitalier français fait face depuis trois décennies à des changements dans son modèle de gouvernance. Ces transformations ont été essentiellement dictées par des politiques publiques souvent porteuses d'injonctions paradoxales mettant à la fois les établissements en concurrence et les incitant à coopérer. Les courants récents de réformes autour de la santé se structurent autour de deux grandes étapes. La première étape, inspirée des thèses du NPM (New Public Management), affirme la nécessité de créer des conditions de marché (quasi marché) en vue d’inciter les hôpitaux à l'efficience (ordonnances de 1996 et T2A ${ }^{1}$ ). La seconde étape, matérialisée par la loi Hôpital, Patients, Santé et Territoires (HPST) de 2009, s’intéresse aux logiques de coopérations entre les hôpitaux. La matérialisation en est les groupements hospitaliers de territoires (GHT). Pour autant, ces deux réformes successives placent les établissements publics et privés dans une situation paradoxale, entre coopération et concurrence, générant des dilemmes complexes et questionnant le positionne-

\footnotetext{
${ }^{1}$ Tarification à l'Activité
} 
ment de ces établissements par rapport à des politiques publiques qui se succèdent en ne convergeant que partiellement. Leur expression et leur déclinaison dans les organisations n’a été que finalement peu explorée. Comment de telles politiques publiques sont-elles mises en œuvre dans les organisations?

La littérature en management public traite la question de l'expression des politiques publiques (Politt et Bouckaert 2011, Gibert 2008) notamment à travers leurs effets paradoxaux ou déviants dans les organisations (Mazouz et al., 2012). La littérature sur la fabrique des politiques publiques présentent, elle, des analyses portant essentiellement sur les phases de conception ou d'évaluation des politiques en se focalisant soit sur les actions (sociologie de l'action, théorie de la diffusion), soit sur les institutions (Institutionnalisme sociologique) contribuant à la construction de ces politiques. En revanche, ces analyses ont délaissé la «boite noire » que constitue la mise en acte des politiques publiques et qui nécessitent d'aborder la question du fonctionnement (Muller, 2000, p. 192) plus que celles des déterminants.

A cet égard, la proximité constitue une variable régulièrement convoquée aujourd'hui pour paramétrer les manières dont doivent fonctionner les politiques publiques. Ce terme proximité largement utilisé dans le discours politique semble paré de toutes les vertus (Lefebvre, 2004) : il permet de donner le sentiment de réduire la distance qui sépare élus et électeurs, de s’afficher proche du local. Les expressions de police de proximité, de politiques urbaines de proximité, culture de proximité, justice de proximité, etc. (Warin, 2004) font référence à la souplesse dans l'action, l'accessibilité aux usagers et la médiation sociale. Toutefois, Le Bart et Lefebvre (2005) s’interrogent sur la consistance de ce terme qui apparait comme une référence obligée voire une incantation sans véritable contenu théorique. Ce travail s’attache à donner un contenu théorique et empirique au terme de proximité et à analyser les effets de celle-ci sur le fonctionnement des politiques publiques.

Pour ce faire, nous mobilisons les travaux de l'école de la proximité, qui s’est intéressée à la question de l'exécution des politiques publiques à travers la notion de gouvernance publique qui consiste à gérer des ressources comprises comme des biens publics (Zimmerman, 2005 ; Gilly et Wallet, 2005). L'approche par les proximités (Gilly et Torre, 2000 ; Boschma, 2005 ; Torre et Rallet 2005 ; Talbot, 2008) cherche à rendre compte des effets de l'espace géographique, des valeurs, d'institutions, des liens sociaux, des connaissances communes, ou encore de structures organisationnelles sur les interactions. La grille de lecture autour de cinq formes de proximité (cognitive, organisationnelle, sociale, institutionnelle et géographique) (Boschma, 2005) largement répandue permet de décrire les rapports entre l'espace, les acteurs et les organisations : elle autorise une lecture dynamique des interactions participant au fonctionnement des politiques publiques car la proximité présente des effets «politiques » qui structurent les manières dont s’exécutent ces politiques. Ces effets «politiques » de la proximité définissent des compromis qui vont se construire entre les acteurs, les politiques et les organisations qui façonnent, plus que les annonces de leurs 
concepteurs, le fonctionnement des politiques publiques et leurs perceptions par les usagers. Ainsi, au-delà la conception des politiques, l'exécution des politiques publiques est envisagée ici comme une phase essentielle de la résolution des problèmes publics. Elle est appréhendée à partir de la grille proxémiste mobilisée ici dans une perspective interne, explorant les relations entre différentes catégories d'acteurs au sein d'une même organisation. Cet article se donne alors pour objectifs de répondre à deux questions : d’une part, définir les effets politiques des proximités sur l'exécution des politiques publiques et, d'autre part, tester empiriquement le cadre conceptuel préalablement défini. Pour ce faire, nous organisons cet article en en trois temps. Dans un premier temps en revenant sur la littérature de la fabrique des politiques publiques, nous préciserons en quoi le cadre conceptuel de l'école de la proximité peut permettre d'analyser les interactions entre les acteurs, les politiques et les organisations qui se nouent au cours de la mise en œuvre des politiques publiques et préciserons la nature des effets politiques des proximités. Dans un deuxième temps, l'étude du cas d'un Centre de Lutte Contre le Cancer (CLCC) nous renseigne sur la manière dont les proximités impulsent les interactions entre les médecins, dirigeants, personnels soignants et personnels support et concourent à la mise en œuvre des politiques publiques de santé dans cet établissement. Enfin, dans un troisième temps, nos principaux résultats empiriques sont discutés.

\section{Les effets du contexte géographique et social dans la fabrique des politiques publiques \\ a) Quelles prises en compte du contexte ? Des approches encore hétérogènes et dispersées}

Nombreuses sont les approches qui soulignent l'intérêt de la prise en compte du contexte dans lequel s’exécutent les politiques publiques. Les approches systémiques de la construction des politiques ont insisté sur la réceptivité des autorités publiques aux signaux ou aux exigences de l'environnement social (Easton, 1974 ; Deutsch, 1966). Cet ensemble de travaux souligne la contingence que crée cet environnement dans la construction des politiques publiques et qui limite dès lors l'éventail de choix des gouvernants et privilégie la reconduction de choix passés (Delpeuch, 2009). Les approches de l'apprentissage des politiques publiques (Bennett et Howlett, 1992) insistent quant à elle sur la prévisibilité des choix de politiques publiques à travers des modes de pensée ou des représentations communes à un ensemble de décideurs. Les approches structurales (Dezalay et Garth, 2002), à l'échelle internationale, et les théories de la diffusion des politiques publiques (Rogers, 2003, Levi-Faur, 2005), plus au niveau national, postulent l'existence de « suivisme » et de « contagion » dans la fabrique des politiques publiques et justifient ces deux phénomènes par l'existence de proximités soit géographiques, soit socio-économiques favorisant la diffusion (Delpeuch, 2009). Enfin, les policy transfer studies (Dolowitz et Marsh, 2000 ; Bulmer et Padgett, 2004 ; Bulmer, 2007) représentent le courant de synthèse des approches rationalistes et sociologiques le 
plus structuré par son aptitude à rapprocher des éléments à la fois macro et microsociologique dans la justification des choix de politiques publiques. Ainsi, des facteurs structurels (institutions, idées, valeurs, culture,...) mais aussi individuels (jeux d'acteurs, apprentissage,...) sont mis au jour par ce courant pour justifier ces choix. Ceci permet de prendre en compte à la fois l'institution sociale et l'interaction entre les acteurs (Delpeuch, op.cit.) dans le fonctionnement des politiques publiques. En revanche, les manières dont ces facteurs s’insèrent dans des processus plus larges de fonctionnement des politiques publiques restent assez peu analysées (ibid.).

\section{b) Structurer la prise en compte du contexte : un passage utile par la proximité}

Ces différentes approches ont pour point de commun d'évaluer les politiques publiques à l'aune de la distance existant entre les acteurs publics et les usagers et les acteurs publics eux-mêmes. Ces approches réussissent, par leur focalisation sur la distance, à déterminer avec justesse les raisons structurelles, institutionnelles ou contextuelles qui justifient des choix de politiques publiques. Celles-ci ne permettent pas d'analyser ces distances autrement que dans une visée descriptive ou compréhensive et de prescrire des politiques en mesure de réduire ces distances. Une analyse inverse en termes de proximités peut à nos yeux enrichir ces analyses en mettant en avant des facteurs en mesure de justifier des choix de politiques publiques. Il s’agit de s’interroger plus sur le « Comment » des politiques publiques plutôt que sur le « Pourquoi » afin de mettre en lumière des éléments qui peuvent mieux circonscrire l'ambivalence du terme proximité en matière de politique publique. Les analyses en termes de proximités géographiques mais aussi sociales, institutionnelles, organisationnelles ou cognitives (Boschma, 2005) en offrant une lecture dans l'espace et dans le temps des interactions entre les acteurs intègrent dans leurs analyses les moments de contact entre des acteurs mais aussi entre leurs systèmes de représentation.

\section{L'Ecole de la proximité}

L'Ecole de la proximité s'intéresse aux conditions de l'apparition et au renforcement d'une interaction entre des acteurs a priori hétérogènes. Historiquement, elle s'est construite au milieu des années quatre-vingt-dix et regroupait des auteurs qui cherchaient à intégrer dans l'analyse économique le rôle de l'espace géographique dans la construction des relations économiques. Très vite, si l'espace est d'abord inséré dans l'analyse dans sa dimension géographique, il le sera dans sa dimension sociale (Bellet et al.,, 1993). Ce courant pose comme principe fondamental que la localisation dans un espace géographique et social influent sur les interactions entre les acteurs (Gilly et Torre, 2000; Boschma 2005).

\section{a) Les dimensions de la proximité}


Les travaux sur la proximité ne définissent pas la proximité par elle-même, mais à travers ses dimensions. L’approche par la proximité est une grille de lecture qui se nourrit de divers courants théoriques (institutionnalisme, interactionnisme, évolutionnisme, théorie de la régulation, théorie des organisations, etc.) pour poser la question de l'influence de la localisation des acteurs sur leurs interactions économiques. Deux types de localisation sont traditionnellement envisagés : la localisation dans l'espace géographique et la localisation dans l'espace social. Dès lors, si le nombre de dimensions distinguées varie selon les auteurs (cf. par exemple pour une synthèse Carrincazeaux, Lung et Vicente, 2008), tous proposent une grille d'analyse fondée sur au moins deux types de proximité, géographique et non géographique.

La proximité géographique se réfère à la question de la position dans l'espace géographique des acteurs, qu'ils soient des individus ou des organisations (Torre et Gilly, 2000). La proximité géographique renvoie à la distance kilométrique qui sépare deux ou plusieurs acteurs. Elle se mesure en intégrant dans l'analyse deux catégories d'effets :

- d'une part, des effets objectifs tels que le coût temporel, de communication et monétaire du franchissement de la distance ;

- d'autre part, un effet subjectif puisqu'elle relève en dernier ressort d'un jugement porté par les individus sur la nature de la distance qui les sépare.

La définition de la proximité non géographique fait débat dans la littérature. La proximité organisée se définit chez Torre et Rallet (2005) par la capacité qu’offre une organisation de faire interagir ses membres. Cette capacité résulte à la fois d'une logique de similitude entre des représentations et d'une logique d'appartenance à une organisation. La logique de similitude exprime la communauté de croyances et de savoirs que partagent les participants et qui les lient ; la logique d'appartenance traduit le fait que les membres d'une organisation interagissent effectivement grâce et dans un même cadre de règles et de routines de comportements dont ils partagent les mêmes interprétations. Boschma (2005), adoptant une perspective évolutionniste, distingue quant à lui quatre proximités non géographiques. L’appartenance à un même arrangement institutionnel (hiérarchie, réseau, marché) au sens de Williamson (1985) qualifie la proximité organisationnelle. Elle évalue le degré d'autonomie juridique et économique existant entre les membres d'une organisation ou entre des organisations. Son existence permet de réduire l'incertitude inhérente à toute relation et l'opportunisme des agents. La proximité cognitive renvoie au partage par les organisations d'une même base de connaissances qui prennent par exemple la forme de brevets ou de publications lorsqu'elles sont codifiées. Similaires et/ou complémentaires, elles ouvrent la voie à une compréhension mutuelle. Cette proximité est nécessaire pour que les firmes puissent absorber de nouvelles connais- 
sances. Se plaçant au niveau individuel, la proximité sociale mesure l'appartenance des individus à un même réseau social au sens de Granovetter (1985). L’encastrement des relations économiques dans un réseau social crée de la confiance entre les acteurs, fondée sur l'amitié, la parenté ou l'expérience passée. Ainsi le risque de conflits en est diminué. Enfin, à un niveau plus collectif, la proximité institutionnelle concerne le partage de diverses institutions plus ou moins formelles comme des lois, des règles, des coutumes, des valeurs, etc. (Kirat et Lung, 1999). Ces institutions fournissent un cadre stable aux relations inter-individuelles et inter-organisationnelles.

De façon générale, l'existence de proximités ne garantit pas qu'une interaction va se nouer entre deux acteurs : il s’agit bien d’une réponse au problème de la séparation, une possibilité offerte aux acteurs, des ressources pour l'action collective encore latente (Talbot, 2008). Pour que ces ressources soient activées, il est nécessaire que les acteurs s’entendent autour d’un projet collectif comme par exemple mettre en œuvre des politiques publiques.

\section{b) Une approche d'abord cognitive}

Que faut-il partager pour agir ensemble ? Très vite il apparaît dans les définitions précédentes que le partage de représentations, qui ont pour première fonction cognitive de comprendre et d'expliquer la réalité, est une condition à toute coordination. Comme l'incertitude envahit toute action, chacun trouve une réponse en se dotant d'images du monde, en attribuant un sens aux informations reçues. Ce processus cognitif est une façon de surmonter en partie l'incertitude et va permettre à chacun de de continuer à agir. Et s’il faut agir collectivement, alors il faut nécessairement partager des représentations.

Dès lors on comprend aisément que les travaux en sciences cognitives ont fait écho au sein de l'école de la proximité. En particulier, l'interactionnisme qui place au centre de l'analyse une représentation particulière essentielle pour un acteur, celle des conséquences de ses actions. Chacun se représente les conséquences de ses actions, celles des autres, puis agit en conséquence. La réciprocité des perspectives est alors une condition au fait d’agir socialement (Le Breton, 2004). Ainsi, pour l'école de la proximité, l'existence d'un cadre cognitif commun devient une condition à l’émergence des interactions. Kirat et Lung (1999) définissent la proximité institutionnelle comme la capacité des acteurs à se coordonner par la convergence de leurs représentations vers un ensemble de règles partagées. La proximité organisée renvoie à la capacité d'une organisation de faire interagir ses membres (Torre, Rallet, 2005). Cette capacité résulte à la fois d'une logique d'appartenance à une organisation et d’une logique de similitude entre des représentations.

Ces travaux s’inscrivent clairement dans une perspective interactionniste qui fait de la proximité un lien social de nature principalement cognitif. Les travaux traitent alors des questions de traitement de l'information, de représentations, de production et de transfert de connaissances. Cette lecture 
traite finalement des conditions d'apparitions d’interactions apaisées, basées sur l'échange, une compréhension mutuelle. Mais elle élude, sans toutefois les nier, le fait que les interactions sont aussi fondées sur des rapports de pouvoir, des conflits, entre des acteurs hétérogènes dans leurs dotations en ressources, ayant des intérêts a priori divergents. On peut alors adopter une lecture complémentaire de la proximité, dite politique.

\section{c) Une approche politique de la proximité}

Que faut-il, au-delà du partage, réguler pour agir ensemble ? Les questions d'ordre politique ${ }^{2}$ de construction de compromis et de choix collectifs, de régulation des conflits, d’imposition de décisions collectives font appel à l'exercice d'un pouvoir, à des relations d'autorité et d'obéissance (Braud, 2008). Les interactions ont ici pour objectifs de permettre aux acteurs d'orienter les choix collectifs et satisfaire ainsi leurs intérêts. Il ne s’agit plus seulement d’interagir pour échanger des connaissances mais aussi pour effectuer un travail politique pour stabiliser ou faire évoluer l'ordre en place (Talbot, 2008). Ce travail politique peut s'entendre comme «l'art de s'arranger avec autrui » (Dubet, 2009, pp. 298-299). Il devient une tentative des acteurs de faire prévaloir une organisation sociale conforme à leurs intérêts, de mettre en œuvre une capacité d’influence sur les choix publics, par exemple en définissant les priorités (Amable, Palombarini, 2004). Il explique la stabilité et la reproduction d'un système social régulé, malgré les contradictions dont il est rempli (Commons, 1934).

Comment les proximités favorisent-elles ce travail politique? Au moins trois types d'effets sont observables. Premièrement, les proximités permettent de réguler les conflits par la confiance. En effet, la pérennité d'une interaction dépend de la capacité des acteurs à réguler leurs conflits : la confiance devient un ingrédient essentiel de cette régulation. Or les proximités, dans leurs diverses dimensions, génèrent une telle confiance. Ainsi, selon Nilsson et Mattes (2015) la proximité géographique constitue un accélérateur de confiance. Partager un même espace géographique permet aux individus d'interagir plus fréquemment en face en face (Bellet et al., 1993 ; Kirat et Lung, 1999 ; Knoben et Oerlemans, 2006). Ce dernier joue un rôle essentiel dans la construction de la confiance en facilitant l'échange d'émotions, de sentiments. En outre, l'espace (ici en fonction de la localisation des acteurs) joue alors un rôle dans la démarche de typification (Lagroye, François, Sawicki, 2006). Se réclamer d'un lieu revient donc à se réclamer d'un groupe social, par association, le tout développant un sentiment de loyauté (Alvesson et Lindkvist, 1993) et d'empathie (Giddens, 1987) et au final de confiance. Toujours dans ce registre, selon Dupuy et Torre (2004), la proximité organisationnelle génère de la confiance car, parce qu’on appartient à une organisation, on en ap-

\footnotetext{
2 Selon Braud (2008, p. 16), «le politique renvoie à ce champ social dominé par des conflits d'intérêts régulés par un pouvoir lui-même monopolisateur de la coercition légitime ». Chez Lagroye, François et Sawicki, (2006), l'espace politique est un domaine d'activité spécialisé qui se signale par la prétention qu’ont certains acteurs d'user d'un droit de commander les membres d'un groupe social.
} 
plique les règles entendues les réponses à apporter à des situations préalablement définies. Toute structure de gouvernance limite les risques d'opportunisme (Williamson, 1985) et l'autonomie juridique et économique du chacun. Enfin, la proximité sociale qui lie par exemple deux individus par la parenté et l'amitié favorise la construction d’une confiance (Granovetter, 1985).

Deuxièmement, les proximités peuvent réguler une relation par le contrôle. Selon Hodgson (2006), les institutions sont des « systems of established and prevalent social rules that structure social interactions. [...] Generally, institutions enable ordered thought, expectation, and action by imposing form and consistency on human activities » (p. 2). Elles ne sont donc pas principalement conçues pour résoudre des problèmes de coordination entre des agents égaux aux intérêts identiques, mais comme des solutions à des conflits entre des acteurs inégaux aux intérêts divergents (Hodgson, 2003). Ces mêmes institutions, parce qu'elles font autorité, ont une fonction stabilisatrice qui se construit par un processus de naturalisation (Douglas, 1986). Du point de vue des acteurs, les institutions sont vécues comme détentrices d'une réalité propre, comme un fait extérieur et coercitif : ils leur semblent alors impossibles de les changer ou même de les fuir. Les institutions enfin bornent les comportements. Se conformer à des institutions revient à adopter des comportements attendus : elles peuvent alors se comprendre comme des prescriptions sociales dont l'acceptation collective est souvent antérieure à la relation. Ainsi le partage d’institutions favorise un pronostic adéquat du comportement d'autrui, ce qui est très utile pour réaliser un travail politique. En outre, une proximité cette fois cognitive rend accessible et compréhensible les connaissances codifiées et tacites détenues par un acteur, ce qui permet de contrôler les connaissances détenues par chacun. Ce contrôle peut-être utile par exemple pour constater une asymétrie de connaissances, asymétrie exploitable par un acteur dans le sens de ses intérêts propres. Ainsi, au-delà du seul contrôle, les proximités peuvent verrouiller une relation. La proximité géographique entre des acteurs contraint ces derniers : chacun hésite à adopter un comportement opportuniste envers un acteur co-localisés car il plus difficile de tromper un inconnu que son voisin avec lequel on partage un même réseau d'amis, d'anciens étudiants, de parents d’élèves, etc. Nous faisons ici référence au phénomène de clan control (Ouchi, 1979), qui renvoie au contrôle social qu'exerce un réseau sur le comportement de ses membres, contrôle d'autant plus fort qu’il est localisé. La proximité géographique viendrait en quelque sorte verrouiller la relation entre des agents. Une trop forte proximité cognitive peut engendrer des phénomènes de lock in, c'est-à-dire d'enfermement dans une trajectoire de développement d'une industrie, d'un territoire etc. (Boschma, 2005) : cet enfermement peut résulter d'un travail politique des acteurs en position dominante en situation de rente qui réalisent des arbitrages dans le sens de leurs intérêts propres.

Enfin, et troisièmement, la proximité peut légitimer l'action publique. Nous l'avons souligné en introduction, discours et action publiques se sont emparés du terme "proximité » (sans en préciser les dimensions) pour re-légitimer le lien social (Le Bart et Lefebvre, 2005). Se déclarer proche sus- 
cite un consensus transpartisan, permet de mettre l'accent sur la disponibilité, l'écoute, de convivialité, de solidarité et d'authenticité (Houllier-Guibert, 2009). La distance n’est plus comprise comme un gage d’égalité de traitement, d’impartialité, de recul nécessaire mais renvoie à une double idée de cécité et d’inefficacité de l'action publique (Lefebvre, 2004). L’utilisation du terme dans le travail politique permet donc de gagner en légitimité, proximité et légitimité étant dorénavant étroitement associées.

Cette grille de lecture de l'école de la proximité en mettant en évidence ces trois effets politiques peut autoriser une analyse étendue du fonctionnement des politiques. Elle peut permettre de circonscrire les effets politiques de la proximité sur les politiques à au moins à trois niveaux (sur la confiance entre acteurs, sur le contrôle organisationnel et sur la légitimité des politiques). Notre étude de cas est destinée à mesurer l'opérationnalité de cette grille de lecture pour évaluer ces effets dans le cas de politiques publiques engagées en parallèle et souvent de manière contradictoire.

\section{Méthodologie : l'étude de cas}

La nature même de l'objet qui nous occupe impose de fait le recours à des méthodes de nature qualitative afin d'appréhender les interactions entre les acteurs et le potentiel explicatif d'une étude concrète des «proximités » intervenant dans les interactions qui assurent la mise en œuvre des politiques publiques. Pour ce faire nous recourons à l'étude de cas unique. Celle-ci s’avère particulièrement pertinente lorsque les problèmes et objets étudiés sont complexes (Wacheux, 1996), fortement contextualisés et que l'étude s'inscrit dans un champ relativement nouveau (Evrard et al, 2009). Tel est le cas des hôpitaux et des questions de gestion et management qui s’y posent. L'étude de cas permet d'étudier si les connaissances sont ou ne sont pas, compatibles avec l'expérience que le chercheur a de la situation pratique considérée, et si les acteurs considèrent qu'elles leur fournissent des repères utiles pour penser et agir dans cette situation en direction de leurs buts (Avenier et al, 2012).

Ce travail ne prétend pas à l'exhaustivité mais par son analyse il doit nous permettre d'ouvrir un espace de réflexion sur les formes de proximités qui peuvent se nouer concrètement dans la mise en œuvre des politiques publiques qui se veulent justement porteuses de rapprochement entre les acteurs.

\section{a) Présentation du cas}

Les Centres de Lutte Contre le Cancer (CLCC) sont des établissements de santé privés à but non lucratif assurant en France une mission de service public hospitalier dans le domaine de la cancérologie. Créés en octobre 1945, ils sont financés par l'assurance maladie et sont contrôlés par 
le ministère de la Santé. Ils relèvent des mêmes obligations financières et administratives que les établissements publics de santé. Leur organisation pluridisciplinaire permet une prise en charge globale du patient grâce à des moyens en chirurgie, en oncologie médicale, en radiothérapie et cytopathologie. Ils sont liés par des conventions avec les universités et les centres hospitaliers universitaires pour définir une organisation commune pour l'enseignement et la recherche en cancérologie. Outre les missions de recherche, d'enseignement et de soins, ils assurent des missions de prévention. Leur direction est assurée par un directeur, médecin, nommé pour cinq ans assisté d'un secrétaire général et d'une équipe de direction marquée par une diversité de cultures professionnelles (gestionnaire, médicale, soignante, etc.).

Le Centre Jean PERRIN de Clermont Ferrand, fondé en 1973, est un des 18 centres de lutte contre le cancer de la fédération nationale UNICANCER. Reconnu comme pôle d'excellence dans sa spécialité, il entretient depuis son ouverture une relation ambivalente avec le CHU avec lequel il partage un même site, la prise en charge régionale de la cancérologie (soins, recherche et enseignement) ainsi que la prise en charge spécialisée sur les centres hospitaliers de périphérie. De fait, si une coopération existe, les deux établissements n’ont jamais développé de projet institutionnel ou médical commun. Depuis le début des années 2010, ces deux établissements sont parvenus à réaliser une répartition des activités chirurgicales et médicales. Par ailleurs, sur le site clermontois, un groupe de cliniques privées constitue un concurrent non négligeable sur une partie des activités en cancérologie, en particulier sur le dépistage. Ainsi, l'évolution de l'environnement (groupements hospitaliers de territoire, contrainte financière, fusion d'établissements) conduits les établissements médicaux à penser de plus en plus en termes de coopération. Dans ce contexte, nous pouvons supposer que la question des interactions entre les personnes, les organisations et les politiques publiques pour mener à bien les transformations nécessaires est un élément particulièrement critique.

\section{b) La démarche de collecte des données}

Des données secondaires (comptes rendus de réunion, présentation du plan stratégique du Centre, organigramme, rapports de gestion) nous ont permis de cerner les principales politiques publiques à l'œuvre dans cet établissement ainsi que les catégories d'acteurs y contribuant. Nous avons notamment participé à l'ensemble des réunions (11 réunions depuis décembre 2015) de réflexion sur la mise en œuvre des politiques de coopération autour des GHT et d'un projet régional d'Institut du Sein.

Nous avons par ailleurs participé avec une équipe de dix responsables du CJP à une visite des Hôpitaux Universitaires de Genève (HUG) qui font fonctionner depuis plusieurs années un institut du Sein. L’ensemble de ces réunions et visites ont fait l’objet de comptes rendus circonstanciés. Les 
Tableau 1 : Les grandes catégories de codage des données secondaires

\begin{tabular}{|c|c|}
\hline Thèmes & Catégories de codage \\
\hline \multirow[t]{2}{*}{$\begin{array}{l}\text { Evolutions } \\
\text { contexte }\end{array}$} & $\begin{array}{l}\text { Contexte réglementaire: encadrement de l'activité (cadre légal, textes } \\
\text { Agence Régionale de Santé et Schéma Régional d’Organisation des } \\
\text { Soins, ...), }\end{array}$ \\
\hline & $\begin{array}{l}\text { Le champ concurrentiel : périmètre d'intervention, positionnements, } \\
\text { ressources et compétences spécifiques des acteurs de la prise en charge du } \\
\text { cancer, }\end{array}$ \\
\hline \multirow{2}{*}{$\begin{array}{l}\text { Transformation de } \\
\text { l’activité du CLCC, }\end{array}$} & Evolution de l'organisation, \\
\hline & Evolution des modes de prise en charge, des parcours de soin, \\
\hline \multirow{2}{*}{$\begin{array}{l}\text { Tensions et } \\
\text { dysfonctionnements, }\end{array}$} & Valeurs et sens de l’action (confiance et légitimité), \\
\hline & $\begin{array}{l}\text { Performance et ses indicateurs (activité, ressources humaines, qualité, } \\
\text { satisfaction) renvoyant à la notion de contrôle, }\end{array}$ \\
\hline $\begin{array}{l}\text { Projets de } \\
\text { coopération et axes } \\
\text { d'innovation }\end{array}$ & $\begin{array}{l}\text { Projets d'établissement, projets de territoire, projets inter-établissements, } \\
\text { Création de nouveaux services, de nouvelles fonctions (case manager), }\end{array}$ \\
\hline
\end{tabular}

La démarche de construction des données primaires s’est appuyée sur ces données secondaires et leur analyse. Les données primaires reposent plus particulièrement sur une série d'entretiens semidirectifs réalisés auprès des responsables de service représentatifs des quatre catégories principales d'acteurs des établissements hospitaliers (dirigeants, médecins, personnels soignants et personnels des fonctions supports) identifiées comme pertinentes à l'issue de l'analyse des informations primaires. Nous avons interviewé pour cela des représentants de chacune de ces catégories. Le tableau 2 représente les caractéristiques des entretiens réalisés.

Tableau 2 : Caractéristiques des personnes interrogées

\begin{tabular}{|c|c|c|c|c|}
\hline $\begin{array}{c}\text { Fonction de la personne } \\
\text { interviewée }\end{array}$ & Catégorie & $\begin{array}{l}\text { Code des } \\
\text { répondants }\end{array}$ & $\begin{array}{c}\text { Ancienneté } \\
\text { dans } \\
\text { l'établissement }\end{array}$ & $\begin{array}{l}\text { Durée de } \\
\text { l’interview }\end{array}$ \\
\hline $\begin{array}{l}\text { Directeur général adjoint de } \\
\text { l'établissement }\end{array}$ & Dirigeant & D1 & 2 ans & 1h 15 \\
\hline $\begin{array}{l}\text { Médecin-chef en charge de la } \\
\text { qualité, }\end{array}$ & Médecin & M1 & 29 ans & 38 minutes \\
\hline $\begin{array}{l}\text { Médecin-chef du département } \\
\text { chirurgie }\end{array}$ & Médecin & M2 & 28 ans & 48 minutes \\
\hline $\begin{array}{l}\text { Médecin en charge de l’unité des } \\
\text { entrées imprévues }\end{array}$ & Médecin & M3 & $\begin{array}{l}5 \text { ans à temps } \\
\text { complet }\end{array}$ & 54 minutes \\
\hline $\begin{array}{l}\text { Cadre de santé du service } \\
\text { imagerie (ancien infirmier) }\end{array}$ & $\begin{array}{l}\text { Personnel } \\
\text { soignant }\end{array}$ & PS1 & 15 ans & 51 minutes \\
\hline $\begin{array}{l}\text { Cadre du département } \\
\text { réanimation (ancien infirmier) }\end{array}$ & $\begin{array}{c}\text { Personnel } \\
\text { soignant }\end{array}$ & PS2 & 19 ans & 58 minutes \\
\hline Directrice des affaires financières & $\begin{array}{l}\text { Fonction } \\
\text { support }\end{array}$ & FS1 & 14 ans & 39 minutes \\
\hline
\end{tabular}




\begin{tabular}{|l|c|c|c|c|}
\hline Informaticien & $\begin{array}{c}\text { Fonction } \\
\text { support }\end{array}$ & FS2 & 35 ans & 57 minutes \\
\hline Secrétaire médicale & $\begin{array}{c}\text { Fonction } \\
\text { support }\end{array}$ & FS3 & 5 ans & 37 minutes \\
\hline
\end{tabular}

Le choix d'interviewer ces personnels s'explique par notre volonté de confirmer et de revenir sur les éléments que nous avions pu recueillir lors des comptes rendus de réunion de mise en œuvre des politiques publiques de santé et de coopération auxquelles nous avions participé. Nous avons fait le choix d'une approche interne et donc d'exclure à ce stade de la recherche l'Agence Régionale de Santé (ARS) chargée de la mise en œuvre de la politique de santé pour nous concentrer sur la manière dont le personnel vivait l'exécution de la politique publique. De même les patients parce qu'il ne contribue que de manière encore périphérique à la mise en œuvre de la politique et s’inscrit plus dans une démarche informative et d'écoute du CLCC vis-à-vis des patients.

Chaque entretien d'une durée de 37 minutes à 1 heure 15 a été réalisé à partir d'un guide d'entretien (Annexe 1) permettant de recenser les différentes proximités vécues par les acteurs dans le cadre de leurs interactions.

Ce guide s'articule autour de trois grands thèmes :

- Les changements en cours ou à venir dans le contexte du Centre Jean Perrin,

- Les interprétations des acteurs relatives à leurs interactions avec les autres acteurs,

- La place des proximités et ses éventuels effets sur la fabrique des politiques publiques.

Les thèmes préalablement identifiés lors de l'analyse des données primaires (contexte réglementaire et concurrentiel, transformations et tensions) constituent une première trame de codage des entretiens destinée à estimer la réalité perçue des évolutions du contexte et des transformations que connaissent les organisations de santé. La seconde trame concerne le codage autour des dimensions de la proximité (géographique, cognitive, sociale, institutionnelle et organisationnelle) de leurs effets (plutôt positifs, neutres ou plutôt négatifs) sur la confiance, le contrôle et la légitimité.

Les entretiens ont été enregistrés et retranscrits pour en faciliter l'analyse de contenu. L'essentiel a consisté en une analyse lexicale manuelle. Celle-ci nous a permis de circonscrire les éléments récurrents justifiant les distances ou les proximités qui peuvent exister entre chaque catégorie d'acteurs dans le cadre de leurs interactions.

\section{Résultats : effets politiques des proximités}

Les analyses des comptes rendus de réunion et des entretiens individuels permettent de mettre en évidence l’importance des dimensions de la proximité et leurs effets ambivalents sur la confiance. Les politiques publiques s'expriment d'abord à travers les relations interindividuelles (dimension cognitive et institutionnelle) solidement ancrées et finalement sur un périmètre géographique relati- 
vement restreint (a), leur appropriation par les acteurs est facilitée par la mise en œuvre de mécanisme de contrôle dont certains sont tacites (b) et d’un mécanisme de gouvernance original (c).

\section{a) Régulation par la confiance}

Lorsqu'est évoquée la possible fusion avec le CLCC de Lyon, les répondants ne voient pas l'intérêt d'une coopération. «La coopération avec Lyon c'est différent d'abord parce qu'il y a une distance, le centre est plus gros avec des contraintes différentes. Le projet dans la configuration qui était proposée a été refusé par la communauté médicale. Il était plus vécu comme une absorption. On ne voyait pas ce que cette collaboration pouvait nous apporter » [M1], «L'important est que le partenaire apporte une chose complémentaire » [PS1]. Si les coopérations résultent d'une véritable volonté stratégique des directions (conserver sa place), elles sont sources de défiance pour les personnels, « les choix faits par la direction sont-ils bien les bons, ne risque-t-on pas de perdre notre activité et nos emplois » [D1], « nos recettes et nos résultats ne doivent pas servir à compenser les déficits du CLLC Lyonnais, on travaille pour les patients de notre région (Auvergne)» [FS1]. La coopération est perçue par certains comme un outil de rationalisation économique qui pourrait à terme réduire l'activité du CLCC en particulier des fonctions support. Pour les soignants son intérêt doit être avant tout de servir les patients. Mais en même temps, la mise en œuvre de coopérations s’impose à l'heure des groupements hospitaliers de territoire. Si elles s’inscrivaient dans une démarche plus volontaire avant l'arrivée des GHT, elles apparaissent désormais comme une quasi obligation : « avec les GHT, on ne pourra pas rester seuls » [FS1], la coopération entre les acteurs de santé d'un territoire devenant finalement une norme.

Ces coopérations sont directement liées la proximité sociale. En effet, «la cancérologie est un petit monde » [M1], « on se connait tous » [M3], car tous les médecins sont passés un jour où l'autre pas le Centre Jean Perrin au cours de sa formation. Ainsi les coopérations sont plus de nature interindividuelle qu'inter-organisationnelle : adressage de patients ne s’inscrivant pas nécessairement dans le cadre de conventions, consultations avancées. Par ailleurs, la proximité sociale permet de compenser la distance géographique, en parlant des coopérations médicales entre les médecins du CLCC et des hôpitaux périphériques : «On se connait bien même si on n’est pas très proches géographiquement. » [M1]. Cependant, sur un autre registre, la proximité géographique au sein de l'établissement qui s'exprime dans une dimension quasi physique apparait pour un répondant comme un passage obligé à la confiance : "il faut occuper le terrain, les gens doivent vous voir, c'est une source de confiance. » [PS2].

Mais en même temps, dans cet établissement, concernant l'exécution des politiques publiques, il existe des proximité cognitive, institutionnelle et organisationnelle qui se créent entre les personnels médicaux d'un même service ou de services partenaires qui n’altère pas pour autant la confiance 
avec les autres personnels (dirigeants ou personnels support). Un médecin explique «si avec les personnels support nous avons un langage très différent, une façon différente de concevoir les choses différente, donc on est obligé de s'expliquer réciproquement, mais cela ne nuit pas à la confiance » [M3] ; «je ne suis pas sûr que la formation que l'on a rapproche les individus, je ne vais pas discuter de la même manière avec un médecin, une aide-soignante mais cela n’altère pas la confiance » [M3].

Ainsi, dans cet établissement de santé, il apparait de façon assez claire que la proximité géographique constitue aux yeux des acteurs un préalable essentiel à la coopération et à la confiance («On a une coopération naturelle avec le CHU parce qu'on est sur le même site » [M2]). Ceci crée un effet par la confiance qui est amplifié par l'existence de proximités sociales entre notamment les médecins qui se connaissent pour avoir suivi les mêmes formations, souvent au Centre Jean Perrin, ou pour participer aux mêmes congrès médicaux. Cette articulation entre proximité géographique et sociale est impulsée par les médecins qui perçoivent un intérêt à disposer sur le plan professionnel d’un réseau proche spatialement et socialement («Les médecins sont avant tout confrères. Même dans la difficulté ils sauront se soutenir, ce ne sera pas nécessairement le cas pour les soignants » [PS1] ; «La cancérologie est un petit monde, tout le monde se connait, en France comme en Auvergne » [M1]). Ceci facilite leur travail et crée un climat de confiance dans une activité (celle de la cancérologie) qui nécessite de bien connaitre ses interlocuteurs pour accélérer les diagnostics et les prises en charge des patients («avec des médecins libéraux, on fonctionne sur la confiance les uns envers les autres, on a étudié ensemble ce qui contribue à créer de bonnes relations. Quand on a échangé des patients, qu'on a rendu service et que la prise en charge s'est bien passée alors la confiance est là. » [M3]).

Les proximités géographique et sociale apparaissent comme deux socles essentiels des effets sur la confiance entre les personnels. Ceci est particulièrement vérifiable dans des métiers hautement qualifiés qui imposent un travail en réseau toujours plus soutenu afin de développer un faisceau de connaissances adapté à un problème toujours spécifique. Ces résultats sont, dans cet établissement, en conformité avec ceux de l'Ecole de la Proximité qui mettent en évidence le rôle central de ces deux proximités dans la construction de la confiance interindividuelle (Dupuy et Torre, 2004). Nos analyses permettent donc de constater le même phénomène pour l'exécution des politiques publiques Par contre, dans ce cas spécifique des politiques publiques en milieu hospitalier, nous constatons que ces proximités géographique et sociale sont complétées par des proximités cognitive, institutionnelle et organisationnelle inhérentes notamment à la forte spécialisation des services et des métiers (chirurgie, anatomo-pathologie, oncologie, hospitalisation) qui imposent de partager des connaissances, des langages et des valeurs propres à chaque profession sans pour autant créer de la défiance entre les services (« on peut avoir confiance dans quelqu'un dont on est moins proche mais 
dont on sait avec l'expérience que c'est un bon professionnel et humainement estimable... ne nécessite pas de contrôle » [M3]).

\section{b) Régulation par le contrôle}

On perçoit dans le discours l'importance du socle axiologique commun à l'ensemble des répondants même si ceux-ci perçoivent un amenuisement de son importance. Les valeurs soignantes sont un élément de recentrage lorsqu’il existe des tensions où conflits : «quand il y a des dérapages on recentre sur le patient et autour des valeurs soignantes » [PS1].

Dans cet établissement, il apparait que le contrôle organisationnel, notamment dans ses aspects formels, s'opèrent plutôt par les proximités cognitive, institutionnelle et organisationnelle. Cette dernière semble être liée au développement de la compétence et de la professionnalisation des métiers. Elle se traduit par une forme de cloisonnement des services et un allègement du contrôle sur les personnels : «Les personnels sont désormais de vrais professionnels. Ils sont responsables de leurs actes. Notre organisation ne peut fonctionner que parce qu'il y a de la confiance » [PS1], mais en même temps s'instaure une nouvelle forme de contrôle institutionnel. En effet, bien que le thème de la qualité ne figure pas dans le guide d'entretien, il a été très évoqué par tous les interrogés. Si la démarche peut être perçue par certains comme une contrainte (médecins), elle constitue un langage commun entre tous les services qui sont par nature, en raison de la multiplicité et de la spécificité des métiers, très cloisonnés. «La qualité réunit les gens. Facilite les relations entre les services, on se retrouve » [M1]. «On demande de plus en plus aux médecins à être impliqués dans le management mais on n'a pas toujours la formation adéquate. »[M2]. Les démarches qualité conduisent à la mise en place de processus et des normes permettant de réduire les dysfonctionnements et finalement à borner les comportements. Certains répondants ont relevé une forme de paradoxe dans ces pratiques.

Ces résultats mettent en évidence dans le fonctionnement des politiques publiques, le rôle essentiel que jouent les valeurs publiques génériques ("Nous Partageons des Valeurs d'humanisme, sociétale, de service public [FS1]»), mais aussi spécifiques à l'établissement, ici, les valeurs soignantes (" quand il y a des dérapages on recentre sur le patient et autour des valeurs soignantes » [PS1]). Aux yeux des responsables des services, la proximité cognitive semble aussi déterminante pour contrôler l'organisation. Ainsi dans cet établissement, en termes de proximité cognitive, la Qualité est qualifiée d' " OVNI » devenu progressivement un outil commun de communication transversale. ("c'est un langage commun à tout le centre, on parle de la même chose et de la même manière » [PS1]). 
De même, ces résultats révèlent les effets sur le contrôle organisationnel que peut générer l'articulation de ces valeurs publiques avec la responsabilisation et l'autonomie des personnels ( "On n'appelle plus les cadres de santé des surveillants, car on ne surveille plus ce qu'ils font, ils sont professionnels, ils sont responsables de leurs actes » [PS1]). Enfin, il convient de noter qu'aux côtés des proximités cognitive, institutionnelle et organisationnelle « la proximité géographique est fondamentale dans la création de la confiance, car elle favorise les échanges et permet d'apprécier le travail de l'autre » «elle est un élément important d'efficacité » [M2].

\section{c) Légitimer l'action publique}

Le travail politique de la direction de faire admettre l'intérêt des coopérations s'ancre pour les CLCC dans une importante proximité cognitive. En effet, à plusieurs reprises les répondants soulignent un système de fonctionnement très original qui constitue un véritable avantage. Le directeur du CLCC est un médecin assisté d'un gestionnaire. Ainsi, pour les personnels médicaux (médecins et soignants) ce statut de dirigeant médecin contribue à légitimer les décisions prises et à générer de la confiance. Cette situation illustre l'importance de la logique de similitude : « Avec la direction on parle le même langage, qui est vraiment un atout. On partage les mêmes valeurs » [PS1], « Cette particularité facilite les relations car on parle à un confrère, un médecin avant d'être un directeur. Ces fonctions font aussi qu'il a des contraintes et qu'il doit trancher par rapport aux contraintes budgétaires que l'on peut comprendre. La direction est aussi là pour arbitrer» [M1] ; «on parle le même langage, les dirigeants sont des médecins ce qui facilite le dialogue » [M3].

On retrouve la même phrase dans les propos de personnel des fonctions support (affaires financières), « avec le directeur adjoint qui est gestionnaire on parle le même langage » [FS1].

Ainsi cette direction à la fois médicale et gestionnaire facilite l'accès à la connaissance en empruntant un langage médical pour tous les personnels médicaux et un langage gestionnaire et les personnels non médicaux. Cependant, si la légitimité s’appuie essentiellement sur les compétences professionnelles pour la majorité des répondants, l'un d'entre eux se questionne sur la légitimité des médecins dirigeants à la tête des CLCC. Ceux-ci ne disposent pas forcément des compétences managériales et stratégiques nécessaires à la direction de ces établissements dans un contexte économique et concurrentiel qui a beaucoup évolué ce qui «peut pénaliser le niveau de confiance » et questionner sa légitimité sur cette fonction particulière (FS2, PS3).

Ici, nous constatons que les effets de la proximité interfèrent plus spécifiquement sur la légitimité des politiques publiques perçue par les personnels. Ces effets s'obtiennent plutôt par des proximités cognitive et institutionnelle. Les valeurs de la profession apparaissent être des actifs immatériels spécifiques (même si des répondants notent une mutation de ces valeurs (FS1, FS2, PS1) dont le respect par les politiques publiques conditionnent la légitimité de ces dernières («Sur la coopération inter établissements, la seule chose qui compte c'est le patient » [PS1]). La qualité apparait 
également comme une valeur commune à l'établissement car les personnels ont été très impliqués dans sa mise en œuvre et ils partagent aujourd'hui les outils qu'ils ont eux-mêmes élaborés. ( $L a$ qualité crée un langage commun on a tous appris en même temps à gérer et mettre en place la qualité »[M1]). Par ailleurs, la construction ou l'agencement de collaborations organisationnelles qui permettent d'assurer une meilleure prise en charge des patients (institut du Sein, réseaux SSR ${ }^{3}$, réseaux d'HAD ${ }^{4}$ ) sont des moyens pour faciliter la construction de la légitimité des politiques publiques («si on ne fait rien notamment sur le sein on risque d'être en danger » [FS1], «En cancérologie, les filières et les coopérations inter établissements sont essentielles à la réussite de la prise en charge des patients »[M3]).

\section{Discussion}

Nos résultats confirment les effets politiques de chaque forme de proximité préalablement définis. Ils permettent de percevoir ces différentes proximités comme des instruments d'accompagnement des politiques publiques lors de leurs mises en œuvre. Le tableau 3 synthétise les effets politiques de la proximité sur la confiance, le contrôle et la légitimité. Les flèches schématisent les liens identifiés entre ces trois derniers éléments.

Tableau 3. Les effets « politiques » de la proximité : enrichissements

\begin{tabular}{|c|c|c|c|}
\hline Proximités & $\begin{array}{l}\text { La confiance } \\
\text { entre les acteurs }\end{array}$ & $\begin{array}{c}\text { Le contrôle organisa- } \\
\text { tionnel }\end{array}$ & $\begin{array}{l}\text { La légitimité des poli- } \\
\text { tiques publiques }\end{array}$ \\
\hline $\begin{array}{l}\text { Proximité géogra- } \\
\text { phique }\end{array}$ & $\begin{array}{l}\text { Par la proximité des } \\
\text { établissements. Par la } \\
\text { filière médicale. }\end{array}$ & $\begin{array}{l}\mathrm{La} \text { proximité géogra- } \\
\text { phique réduit le contrôle } \\
\text { direct }\end{array}$ & \\
\hline Proximité cognitive & $\begin{array}{l}\text { Par les langages profes- } \\
\text { sionnels et leurs traduc- } \\
\text { tions }\end{array}$ & $\begin{array}{l}\text { Par un langage managé- } \\
\text { rial commun } \\
\text { (gestion de la qualité) }\end{array}$ & $\begin{array}{l}\text { Par les dirigeants qui doi- } \\
\text { vent traduire leurs choix } \\
\text { stratégiques }\end{array}$ \\
\hline $\begin{array}{l}\text { Proximité institution- } \\
\text { nelle }\end{array}$ & $\begin{array}{l}\text { Par les valeurs (soi- } \\
\text { gnantes et humanistes) }\end{array}$ & $\begin{array}{l}\text { Par les valeurs et les pro- } \\
\text { cédures du travail médical }\end{array}$ & $\begin{array}{l}\text { Par les valeurs (soignantes } \\
\text { et humanistes) }\end{array}$ \\
\hline Proximité sociale & $\begin{array}{l}\text { Par les mondes domi- } \\
\text { nants (médecins) }\end{array}$ & & \\
\hline $\begin{array}{l}\text { Proximité organisa- } \\
\text { tionnelle }\end{array}$ & $\begin{array}{l}\text { Par la professionnalisa- } \\
\text { tion des services }\end{array}$ & $\begin{array}{l}\text { Par la responsabilisation } \\
\text { et l'autonomie des per- } \\
\text { sonnels. }\end{array}$ & \\
\hline
\end{tabular}

\footnotetext{
${ }^{3}$ Soins de Suite et de Réadaptation

${ }^{4}$ Hospitalisation à Domicile
} 
Dans un premier temps, comme cela avait été démontré dans les organisations marchandes (Dupuy et Torre, op.cit.), c'est la combinaison de l'ensemble des proximités qui joue un rôle essentiel dans la construction de la confiance pour l'exécution des politiques publiques. Ici, la construction de la confiance se réalise à travers les cinq formes de proximité et pour cela elle met en avant l’importance de valeurs et lieux partagés malgré la spécialisation ou la professionnalisation des services qu'imposent souvent le traitement du cancer (tableau 3). En outre, ce résultat met en valeur les rôles essentiels, dans la construction de la confiance en milieu hospitalier, à la fois de la spécialisation des services mais aussi des nécessaires efforts de traduction et de partage de valeurs soignantes et humanistes.

Deuxièmement, dans notre étude de cas, à côté de la proximité géographique, ce sont plutôt les proximités cognitives, institutionnelles et organisationnelles qui permettent de construire le contrôle organisationnel des politiques publiques. De même, ces trois proximités semblent s’articuler pour faciliter le contrôle organisationnel. En effet, l'apparition de valeurs publiques communes (proximité institutionnelle) et d'un langage dominant (proximité cognitive), assure un contrôle organisationnel fort et autorise une plus grande responsabilisation et autonomie dans le travail des personnels. Ces résultats correspondent aux résultats obtenus par l'école de la proximité (Levy et Talbot, 2015). Ils mettent de plus en évidence dans le fonctionnement des politiques publiques, le rôle essentiel que jouent les valeurs publiques de l'établissement et un langage managérial commun (la qualité). Enfin, troisièmement, nous suggérons que les effets sur la légitimité mis en évidence par notre modèle conceptuel se produisent plutôt au niveau de la légitimité des politiques publiques. L’analyse, par l’Ecole de la proximité, des effets de la proximité sur la légitimité a déjà été menée dans plusieurs travaux et elle portait plutôt sur la légitimité des formes de pouvoir en général (Fligstein, 1996 ; Talbot, 2010). Par son application sur le contexte particulier des politiques publiques, notre travail dégage un résultat original qui met en évidence les rôles essentiels des proximités institutionnelle et cognitive dans la construction de la légitimité publique perçue par les personnels. Ces dernières proximités peuvent être définies comme des éléments en prendre en considération lors de la phase de conception des politiques publiques. Notamment afin de prévoir des règles ou de procédures (proximité institutionnelle) construites avec le personnel et une explication des choix stratégiques (proximité cognitive) en mesure de supporter l'exécution de ces politiques. La participation et la prise d'initiative des personnels pour construire leurs propres dispositifs règlementaires ou cognitifs constituent une originalité de nos constatations. Elles semblent être vécues par les personnels comme une condition essentielle pour les rapprocher des politiques publiques qu'ils mettent en œuvre et ainsi pour renforcer les légitimités de ces politiques.

À côté des effets politiques de la proximité sur l'exécution des politiques publiques mis en avant par notre étude de cas, il apparait clairement que ces effets se développent également de manière indirecte à travers les effets réciproques entre la confiance, le contrôle et la légitimité (tableau 3). Même 
si notre approche méthodologique n’était pas totalement orientée vers la description de ces effets, il nous est possible, dans le cadre limité de notre étude de cas, de décrire quelques exemples de cette réciprocité. Ainsi, comme l'indiquent les flèches du tableau 3, la proximité géographique en permettant de construire une confiance entre les acteurs facilite l'évaluation en réduisant les formes de contrôle direct. Ceci en retour, amplifie la confiance entre les personnels et entre les personnels et leurs supérieurs.

Il en va de même concernant la proximité institutionnelle pour laquelle la confiance crée par les valeurs soignantes et humanistes des personnels se répercutent sur les instruments de contrôle (procédures de travail médical) et les politiques publiques qui reprennent ces valeurs.

Enfin, en matière de de proximité organisationnelle, la confiance créée par la spécialisation et la professionnalisation des services autorise un contrôle organisationnel fondé sur l'autonomie des personnels.

Ces exemples représentent certains effets indirects des proximités sur l'exécution des politiques par l’intermédiaire des relations réciproques entre confiance, contrôle et légitimité. Ainsi, ils confirment l'existence d'effets directs et indirects des cinq proximités (Boschma, op.cit.) sur la mise en œuvre des politiques publiques.

Par ailleurs, cette dernière analyse nous permet de proposer deux résultats complémentaires de notre travail. D’une part, notre étude de cas démontre que la confiance apparait être un élément, à la fois, déterminé directement par l'ensemble des proximités et, de manière indirecte, déterminant dans la construction du contrôle organisationnel et de la légitimité des politiques publiques. D’autre part, le contrôle et la légitimité des politiques se construisent par des effets directs des proximités illustrés plus haut mais dont la survenance est également amplifiée par les effets réciproques que la confiance, le contrôle et la légitimité peuvent avoir les uns sur les autres.

\section{Conclusion}

Notre travail visait à faire de l'exécution des politiques publiques un objet de recherche à part entière. La proximité, souvent convoquée pour concevoir ou évaluer les politiques publiques, dispose également d'un pouvoir explicatif de leur exécution par l'exploration de sa face interne décrite notamment par les objets intermédiaires (Vinck, 2009) que sont les personnels et les organisations en charge de cette exécution.

Notre étude de cas de type monographique a permis d'explorer la situation d'un établissement français de lutte contre le cancer qui, par son statut, réalise des missions publiques transverses appropriées de manière singulière par les personnels. Cette appropriation en se déroulant dans l'action, au cours de la mise en œuvre des politiques publiques, nécessite une lecture spécifique des interactions entre ces personnels, leurs organisations et les politiques en question. 
Dans cette perspective, les apports de notre travail sont doubles. D’une part, sur le plan descriptif et compréhensif, notre travail propose une heuristique d'analyse de ces interactions à partir d'une analyse des proximités entre ces différents objets intermédiaires. Ceci permet sur un plan pratique d'attribuer à plusieurs proximités (géographique, cognitive, institutionnelle, sociale et organisationnelle) une place centrale dans la compréhension des choix qui peuvent s’opérer dans la mise en action des politiques publiques.

Par ailleurs, sur un plan prescriptif, notre article permet de formuler, à partir du cadre de l'École de la proximité, un certain nombre de propositions relatives aux effets directs et indirects de ces proximités sur l'exécution des politiques publiques.

Néanmoins, notre travail présente un certain nombre de limites à la fois méthodologique et conceptuelle. Sur le plan méthodologique, nos conclusions imposeront, dans l'avenir, de porter notre attention sur un échantillon plus étendu de personnels de l'établissement pour confirmer ou infirmer nos propositions. L'ambition de ce premier travail était surtout de mettre en avant, à travers la proposition d'une approche heuristique originale, les apports potentiels de l'analyse par les proximités à l'étude du fonctionnement des politiques publiques. Cet outil analytique devra être éprouvé par la suite dans d'autres contextes d'exécution de politiques publiques.

Sur le plan conceptuel, notre travail teste le modèle de l’École de la proximité sur la mise en œuvre de politiques publiques. Il avait pour ambition de mesurer son opérationnalité dans ce type de contexte. Il apparait que le test est plutôt probant car les résultats de ce modèle sur notre étude de cas semblent être très proches de ceux formulés dans les travaux précédents. Par ailleurs, son application dans un contexte public nous a permis d'élargir les effets de la proximité sur la légitimité des politiques publiques et non pas uniquement sur celle des décisions managériales. Les propositions que nous formulons pour renforcer l'exécution des politiques publiques ne sont que propédeutiques et nécessitent d'être consolidés dans nos travaux à venir. En outre, notre analyse se limite ici aux effets des proximités sur l'exécution des politiques publiques : or la causalité inverse existe aussi, puisqu'une fois les politiques publiques mises en œuvre, les proximités se trouvent modifiées. Par exemple, la proximité organisationnelle peut être renforcée suite à la mise en œuvre de coopérations et la proximité sociale se nourrir des interactions individuelles qui se sont nouées à l'occasion de ces coopérations. Au final, dans un cercle vertueux, la confiance s’en trouve accentuée. Enfin, des liens existent entre les effets eux-mêmes, par exemple entre légitimité et confiance ou entre contrôle et confiance : dans ce dernier cas par exemple, le contrôle formel réduit la motivation à adopter des comportements opportunistes ce qui renforce la confiance. Autant de liens de causalité qui appellent de nouvelles recherches. 


\section{Bibliographie}

Amable B., Palombarini S., 2005. L'économie politique n'est pas une science morale. Raisons d'agir Editions, Paris.

Bellet, M. ; Colletis, G. ; Lung, Y. 1993. Economie de proximités , numéro spécial de la Revue d'Economie Régionale et Urbaine, (3).

Bennett, C.J., Howlett, M.,1992. The Lessons of Learning: Reconciling Theories of Policy Learning and Policy Change, Policy Sciences, 25, (3), 275-294.

Boschma, R.A., 2005. Proximity and innovation. A critical assessment. Regional Studies, vol. 39, $\mathrm{n}^{\circ}$ 1, p. 61-74.

Braud P., 2008. Sociologie politique. 9e édition, Lextenso éditions, Paris.

Bulmer, S., Padgett, S., 2004. +Policy Transfer in the European Union: An Institutionalist Perspective. British Journal of Political Science, 35(1), 103-126.

Bulmer, S., 2007. Germany, Britain and the European Union: Convergence through Policy Transfer, German Politics, 16(1), 39-57.

Carrincazeaux, C., Lung, Y., Vicente, J. 2008. The scientific trajectory of the french school of proximity: interaction- and institution- based approaches to Regional System of Innovation. European Planning Studies, 16 (5), 617-628.

Commons J.R., 1934. Institutional Economics. Its Place in Political Economy. MacMillan Company, New York.

Delpeuch, T., 2009. Comprendre la circulation internationale des solutions d'action publique: panorama des policy transfer studies. Critique internationale, (2), 153-165.

Deutsch K.W., 1966, The Nerves of Government. New York Free Press

Dezalay, Y., Garth, B.G., 2002. Legitimating the New Legal Orthodoxy, in Yves Dezalay, Bryant G. Garth (eds.), Global Prescription. The Production, Exportation, and Importation of a New Legal Orthodoxy, Ann Arbor, University of Michigan Press, p. 307.

Dolowitz, D.P., Marsh, D., 2000. Learning from Abroad: The Role of Policy Transfer in Contemporary Policy Making, Governance, 13(1), 5-24.

Douglas, M. 1986. How Institutions Thinks. Syracuse University Press.

Dubet F., 2009. Le travail des sociétés. Editions du Seuil, Paris.

Dupuy C., Torre, A. 2004. Confiance et proximité, dans Pecqueur et Zimmermann. (Eds), Economie de Proximités, Hermès, Lavoisier, Paris.

Easton D., 1974. Analyse du système politique. Armand Colin, Paris.

Fligstein N., 1996. Markets as politics: a political-cultural approach to market institutions. American Sociological Review 61 (4), 656-673.

Gibert P., 2008. Un ou quatre managements publics ? Politiques et management public, 26 (3), 720.

Giddens A., 1987. La constitution de la société, Presses Universitaires de France, Paris.

Gilly, J.P. ; Torre, A (éds), 2000. Dynamiques de proximité, Paris : L'Harmattan.

Gilly, J.P. ; Wallet, F. 2005. Enchevêtrement des espaces de régulation et gouvernance territoriale.

Les processus d'innovation institutionnelle dans la politique des pays en France, janvier, 1-20.

Granovetter M., 1985. Economic Action and Social Structure : The Problem of Embeddedness, American Journal of Sociology, 91 (3), 481-510.

Hodgson, G. 2003. John R. Commons and the foundations of institutional economics. Journal of Economic Issues 37 (3), 547-577.

Hodgson, G., 2006. What are institutions? Journal of Economic Issues, 40 (1), 1-25. 
Houllier-Guibert C-E, 2009. Evolution de la communication territoriale : les limites de l'idéologie de la proximité, Les Enjeux de l'information et de la communication 1/2009, 45-61

Kirat, T., Lung, Y. 1999. Innovation and proximity. Territories as loci of collective learning processes, European Urban and Regional Studies, 6(1), 27-38.

Knoben, J., Oerlemans, L., 2006. Proximity and inter-organizational collaboration: a literature review, International Journal of Management Reviews 8 (2), 71-89.

Lagroye J., François B., Sawicki F., 2006, Sociologie Politique, 5e édition, Presses de Sciences Po et Dalloz, Paris.

Le Breton D., 2004. L’interactionnisme symbolique. Presses Universitaires de France, Paris.

Le Bart, .C. Lefebvre, R. 2005. La proximité en politique: Usages, rhétoriques, pratiques, Presses Universitaire de Rennes, Rennes.

Lefebvre R., 2004. Quand légitimité rime avec proximité, Mouvements 2 (32) , 135-138

Levi-Faur, D., Vigoda-Gadot, E., 2006. New Public Policy, New Policy Transfers: Some Characteristics of a New Order in the Making, International Journal of Public Administration, 29, 247-262.

Levi-Faur, D., 2005. « The Global Diffusion of Regulatory Capitalism », The Annals of the American Academy of Political and Social Science, vol.598, 2005, p. 26.

Levy, R., \& Talbot, D. 2015. Control by proximity: evidence from the 'Aerospace Valley'competitiveness cluster. Regional Studies, 49(6), 955-972.

Mazouz B., Garzon C. et Picard P., 2012. Les déviances dans les organisations publiques en quête de performance. Vers une gestion prophylactique des risques de deviance. Management International, 16 (3), 92-100.

Muller, P., 2000. L'analyse cognitive des politiques publiques: vers une sociologie politique de l'action publique. Revue française de science politique, 189-207.

Nilsson, M., \& Mattes, J. 2015. The spatiality of trust: Factors influencing the creation of trust and the role of face-to-face contacts. European Management Journal, 33(4), 230-244.

Ouchi, W.G., 1979. A conceptual framework for the design of organizational control mechanisms, Management Science, 25(9), 833-848.

Politt C., Bouckaert, G., 2011. Public Management Reform: A Comparative Analysis. New

Public Management, Governance, and the Neo-Weberian State, Oxford/New York, Oxford University Press.

Rogers, E., 2003. Diffusion of Innovations, New York, The Free Press, 2003, 512 p.

Talbot D., 2008. Les institutions créatrices de proximités, Revue d'Economie Régionale et Urbaine, 3, 289-310.

Talbot D., 2010. La dimension politique dans l'approche de la proximité, Géographie, Economie, Société, 12(2), 125-144.

Torre, A.; Rallet, A., 2005. Proximity and localization , Regional Studies, 39(1), 47- 60

Vinck, D., 2009. De l'objet intermédiaire à l'objet-frontière. Revue d'anthropologie des connaissances, 3(1), 51-72.

Warin P., 2004. La gestion de proximité à l'épreuve de politiques publiques en France. In Jouve, Bernard; Booth, Philip;. Démocraties métropolitaines. Transformations de l'Etat et politiques urbaines au Canada, en France et en Grande-Bretagne, Presses de l'Université du Québec, pp.195215, <halshs-00290222>

Williamson, O.E., 1985. The Economic Institutions of Capitalism. The Free Press, Macmillan: New York.

Zimmermann J.-B., 2005. Entreprises et territoires : entre nomadisme et ancrage territorial, La Revue de l'Ires, (47), 21-36. 